\title{
Ovarian cancer proliferation and apoptosis are regulated by human transfer RNA methyltransferase 9-likevia LIN9
}

\author{
HUAI MEI CHEN, JIA WANG, YING FENG ZHANG and YAN HONG GAO
}

\author{
Department of Obstetrics and Gynecology, \\ University-Town Hospital of Chongqing Medical University, Chongqing 401331, P.R. China
}

Received February 18, 2016; Accepted January 31, 2017

DOI: $10.3892 / \mathrm{ol} .2017 .6750$

\begin{abstract}
Current traditional treatment options have little impact on the long-term survival of patients with ovarian cancer due to a lack of understanding of the molecular transformations that occur in ovarian carcinoma. Transfer RNAs (tRNAs) perform a key role in protein translational fidelity. Enzymes involved in tRNA modification may function as regulators of cancer progression. Human tRNA methyltransferase 9-like (hTRM9L) catalyzes tRNA wobble base modifications, which regulate ovarian cancer growth and apoptosis via the retinoblastoma protein (pRB) and p53 signaling pathways. The aim of the present study was to confirm the role of hTRM9L in the proliferation and apoptosis of ovarian cancer. Immunohistochemistry was performed to investigate the expression of hTRM9L and LIN9 in 70 ovarian tissues. hTRM9L was amplified by polymerase chain reaction (PCR) and inserted into the Ubi-multiple cloning site-enhanced green fluorescent protein (EGFP)-internal ribosome entry site-puromycin lentiviral expression vector to create the Ubi-KIAA1456-EGFP-puromycin (LV-KIAA1456) vector. The lentiviruses were subsequently compounded and transduced into HO8910PM cells. hTRM9L, LIN9 and B-cell lymphoma 2 (Bcl-2)/Bcl-2 associated X protein (Bax) expression levels were examined by PCR and western blot analysis. Apoptosis was verified by flow cytometry, and cell proliferation was evaluated using Cell Counting Kit-8. hTRM9L and LIN9 expression were reduced in the ovarian cancer group, and there was a positive correlation between hTRM9L and LIN9 expression according to Pearson's correlation coefficient $(\mathrm{r}=0.406 ; \mathrm{P}<0.05)$. hTRM9L was increased by 2 -3-foldin HO8910PM cells following LV-hTRM9L transduction. The expression of hTRM9L at the mRNA and protein levels in HO8910PM cells that were transfected with LV-hTRM9L was
\end{abstract}

Correspondence to: Professor Jia Wang, Department of Obstetrics and Gynecology, University-Town Hospital of Chongqing Medical University, 55 University City Road, Chongqing 401331, P.R. China E-mail: 752203031@qq.com

Key words: ovarian cancer, HO8910PM cells, human transfer RNA methyl transferase 9-like, apoptosis significantly increased compared with the negative control, as confirmed by reverse transcription-quantitative PCR and western blot analysis, respectively $(\mathrm{P}<0.05)$.The same was observed for LIN9 and Bax $(\mathrm{P}<0.05)$. By contrast, Bcl-2 was downregulated in LV-hTRM9L $(\mathrm{P}<0.05)$. Furthermore, cell growth was inhibited $(\mathrm{P}<0.05)$ and apoptosis increased $(\mathrm{P}<0.05)$. In the present study, hTRM9L was shown to prevent tumor growth and promote apoptosis by regulating LIN9, which is associated with the pRB and p53 signaling pathways. This maybe a novel breakthrough in the treatment of ovarian cancer.

\section{Introduction}

Ovarian cancer is one of the three most common malignant tumors in the female reproductive system, and its incidence is second only to endometrial cancer (1). Various factors, including chemical, physical and biological carcinogenic factors, genetic factors, immune factors and poor lifestyle choices contribute to the etiology of this disease (2). Although surgical resection and chemoradiotherapy have gradually improved, the survival rates for ovarian cancer remain poor (3), and current traditional treatments have little impact on long-term survival. This may be due to the lack of understanding of the molecular transformations that occur.

Transfer RNAs (tRNAs) are key to protein translation fidelity. Growing evidence indicates that aberrant regulation of translation is a trigger for cell transformation in cancer, and tRNA modification enzymes may function as regulators of cancer progression. Human tRNA methyltransferase 9-like (hTRM9L) catalyzes tRNA wobble base modifications. TRM9 forms a complex with Trm112 and uses the methyl donor S-adenosylmethionine to catalyze the formation of 5-methoxycarbonylmethyluridine [a precursor for 5-methoxycarbonylmethyl-2-thiouridine $\left(\mathrm{mcm}^{5} \mathrm{~s}^{2} \mathrm{U}\right)(4)$ and $\mathrm{mcm}^{5} \mathrm{~s}^{2} \mathrm{U}$, which serve important roles in the formation of tRNA ${ }^{\mathrm{Arg}}$ and tRNA ${ }^{\text {Glu }}(5)$, respectively. In the absence of tRNA ${ }^{\mathrm{Arg}}$ and $\operatorname{tRNA}^{\mathrm{Glu}}(6)$, the resulting translational infidelity promotes protein errors and triggers a cascade in the retinoblastoma protein (pRB) and p53 signaling pathways (7).

In humans, the hTRM9L gene maps to the end of human chromosome 8 , a region that is commonly silenced or lost in numerous types of cancer (8). In a previous study by Begley et al (9), the level of hTRM9L in human cancer was 
detected by a panel tissue array, which demonstrated significant downregulation of hTRM9L in ovarian cancer, with an average change of 1.5-fold; the downregulation of hTRM9L was most pronounced in stage IV cancer (10). Therefore, in the present study, it was hypothesized that hTRM9L may serve a role in controlling ovarian cancer development. In the present study, low hTRM9L expression was observed in ovarian cancer tissues, as well as slowed growth of HO8910PM cells with stable transduction of hTRM9L. By contrast, apoptosis was increased. The mechanism of action may involve the activation of the pRB signaling pathway by hTRM9L via LIN9, thereby inhibiting cell proliferation and driving the p53 pathway to promote apoptosis.

\section{Materials and methods}

Patients. The present study consisted of 70 ovarian cancer patients who had not undergone chemotherapy, radiotherapy or hormonal therapy in the 3 months prior to surgery, between December 2013 and June 2014. The ovarian cancer specimens were obtained from the Department of Gynecologic Surgery, University-Town Hospital of Chongqing Medical University (Chongqing, China). Department of Gynecologic Surgery at University-Town Hospital of Chongqing Medical University (Chongqing, China). The use of the tissue specimens was approved by the Medical Ethics Review Committee at the University-Town Hospital of Chongqing Medical University and written informed consent was obtained from all patients.

Reagents and antibodies. The human ovarian epithelial cell line HO8910PM was purchased from American Type Culture Collection (Manassas, VA, USA). The following antibodies were purchased for use in the SP-9000IHCkit (Zhongshan Chemical, Beijing, China): Rabbit anti-hTRM9L (1:100; cat. no. bs-17007RS; Beijing Biosynthesis Biotechnology Co., Ltd., Beijing, China); mouse anti-LIN9 (1:200; cat. no. sc-130571; SantaCruz Biotechnology, Inc., Dallas, TX, USA); rabbit anti-B cell lymphoma 2 (Bcl-2; 1:100; cat. no. bs-0032R; Beijing Biosynthesis Biotechnology Co., Ltd.)/Bcl-2 associated X protein (Bax; 1:100; cat. no. bs-0127R; Beijing Biosynthesis Biotechnology Co., Ltd.); HRP-conjugated goat anti-rabbit immunoglobulin (IgG; cat. no. ZB-230; OriGene Technologies, Inc., Beijing, China); $\beta$-actin (1:5,000; cat. no. BC007330; Wuhan Sanying Biotechnology, Wuhan, China); anti-rabbit IgG (1:1,000; cat. no. 0007-2; Wuhan Sanying Biotechnology) and anti-mouse IgG (1:1,000; cat. no. 00007-1; Wuhan Sanying Biotechnology).

Immunohistochemistry (IHC). Staining was performed with the IHC SP-9000 kit (cat. no. SPN-9001; OriGene Technologies, Inc.) to detect hTRM9L and LIN9 expression levels. Slides were deparaffinized, and antigens were retrieved by heating in a microwave oven for $10 \mathrm{~min}$ at $100^{\circ} \mathrm{C}$ in citrate buffer, followed by incubation in $3 \%$ hydrogen peroxide for $10 \mathrm{~min}$ and blocking with normal goat serum (cat. no. SPN-9001; OriGene Technologies, Inc.) for $20 \mathrm{~min}$ at $20^{\circ} \mathrm{C}$. Slides were incubated with primary antibody targeting hTRM9L (1:100; cat. no. bs-17007RS; BIOSS, Beijing, China) overnight at $4^{\circ} \mathrm{C}$, rewarmed for $30 \mathrm{~min}$ at $37^{\circ} \mathrm{C}$ and then incubated with anti-rabbit IgG secondary antibodies (1:100; cat. no. SPN-9001;
OriGene Technologies, Inc.) at $37^{\circ} \mathrm{C}$ for $30 \mathrm{~min}$. The slides were then incubated with streptavidin-horseradish-peroxidase (HRP) for $20 \mathrm{~min}$ at $37^{\circ} \mathrm{C}$, rinsed with PBS, incubated for 15 min with 3,3-diaminobenzidine (Bopei Biotech Co., Ltd., Chongqing, China) and counterstained with hematoxylin. The staining results of the targeted proteins were observed under a transmission light microscope (magnifications, x200 and $\mathrm{x} 400$ ). Negative controls were prepared using PBS in place of the primary antibody.

Cell culture. The cells were cultured in RPMI-1640 (HyClone; GE Healthcare Life Sciences, Logan, UT, USA) containing $10 \%$ fetal bovine serum (FBS, HyClone; GE Healthcare Life Sciences) in a humidified $5 \% \mathrm{CO}_{2}$ atmosphere at $37^{\circ} \mathrm{C}$. Cell growth was observed using an inverted microscope at a magnification of $\mathrm{x} 10$. Once the cells were grown to $80-90 \%$ confluence, they were digested with $0.125 \%$ trypsin. The medium was replaced every day, and cells were passaged every 2-3 days. Cells in the logarithmic phase were selected for subsequent testing.

Lentiviral overexpression of hTRM9L in HO8910PM cells. The cDNA for hTRM9L (NM_020844) was cloned into pIRES2 by polymerase chain reaction (PCR; Invitrogen; Thermo Fisher Scientific, Inc., Waltham, MA, USA) via homologous recombination between pIRES2 and Ubi-multiple cloning site-enhanced green fluorescent protein (EGFP; GeneChem Co., Ltd., Shanghai, China). Lentiviral vector plasmids for overexpressing hTRM9L were obtained and lentivirus particles were subsequently packaged according to the manufacturer's protocol (Invitrogen; Thermo Fisher Scientific, Inc.). An empty vector lentivirus gene delivery system was used as a negative control. HO8910PM cells were seeded onto 24-well plates at a concentration of $1 \times 10^{5}$ perwell (50-60\% confluence) on the day prior to transfection. LV-hTRM9L was transduced into cells at a multiplicity of infection of 50 using polybrene $(10 \mu \mathrm{g} / \mathrm{ml})$ and enhanced infection solution (GeneChem Co., Ltd.). A non-target virus LV-EGFP (GeneChem Co., Ltd.) was transduced into cells as a negative control. The enhanced infection solution was replaced with RPMI-1640 medium supplemented with $10 \%$ FBS following incubation for $12 \mathrm{~h}$. The transfection validity was detected $72 \mathrm{~h}$ following transfection.

Reverse transcription-quantitative PCR (RT-qPCR). Total RNA was extracted from the cultured cells using TRIzol (Invitrogen; Thermo Fisher Scientific, Inc.), according to the manufacturer's protocol. The concentration and purity of RNA were quantified using a UV spectrophotometer (UltroSPec2100Pro; GE Healthcare Life Sciences). Total RNA was reverse transcribed using the PrimeScript RT reagent kit (Takara Biotechnology Co., Ltd., Dalian, China) in a total volume of $20 \mu \mathrm{l}$, according to the manufacturer's protocol. RT-qPCR was performed using SYBR Premix Ex Taq II (Takara Biotechnology Co., Ltd.) as follows: 1 cycle at $95^{\circ} \mathrm{C}$ for $30 \mathrm{sec} ; 40$ cycles at $95^{\circ} \mathrm{C}$ for $5 \mathrm{sec}$ and $60^{\circ} \mathrm{C}$ for $20 \mathrm{sec} ; 1$ cycle at $65^{\circ} \mathrm{C}$ for $15 \mathrm{sec}$. GAPDH was used as an internal control. Relative mRNA expression levels were analyzed using the $2^{-\Delta \Delta \mathrm{Cq}}$ method (11). The primer sequences are listed in Table I. 
Table I. Primer sequences.

\begin{tabular}{lc}
\hline Gene name & Primer sequence \\
hTRM9L & \\
Forward & 5'-CCGGAGGCCAACTGATGATTT-3' \\
Reverse & 5'-CAGAACAGCTACACTCAGAGC-3' \\
LIN9 & \\
Forward & 5'-GGAACGAAAGTTACAGCACGA-3' \\
Reverse & 5'-CAAGCCCTGTCCTATCAAAAGT-3' \\
Bax & \\
Forward & 5'-TTGCTTCAGGGTTTCATCC-3' \\
Reverse & 5'-GACACTCGCTCAGCTTCTTG-3' \\
Bcl-2 & \\
Forward & 5'-GGCCTCTGTTTGATTTCTCC-3' \\
Reverse & 5'-GCAGGCATGTTGACTTCACT-3' \\
GAPDH & \\
Forward & 5'-ACCACAGTCCATGCCATCCA-3' \\
Reverse & 5'-TCCACCACCCTGTTGCTGTA-3' \\
\hline
\end{tabular}

hTRM9L, human transfer RNA methyltransferase 9-like; Bcl-2, B-cell lymphoma 2; Bax, Bcl-2 associated X protein.

Western blot analysis. Cells in the logarithmic growth phase were harvested. Total protein was then extracted from the cells using radio immuno precipitation assay lysis buffer according to the manufacturer's protocol (KeyGen Biotech Co., Ltd., Nanjing, China). Following centrifugation at 16,099 $\mathrm{x} g$ at $4^{\circ} \mathrm{C}$ for $15 \mathrm{~min}$, the supernatants of the lysates were collected for use. The protein concentration was quantified using a bicinchoninic acid protein assay kit (Beyotime Institute of Biotechnology, Haimen, China). The indicated protein amounts $(50 \mu \mathrm{g} /$ well) were separated by $10 \%$ SDS-PAGE and transferred to polyvinylidene difluoride membranes (EMD Millipore, Billerica, MA, USA). The membranes were blocked with $5 \%$ bovine serum albumin (Beyotime Institute of Biotechnology) for $2.5 \mathrm{~h}$ at room temperature and subsequently incubated with the following primary antibodies overnight at $4{ }^{\circ} \mathrm{C}$ : Rabbit anti-hTRM9L (dilution, 1:500); rabbit anti-LIN9 (dilution, 1:100); rabbit anti-Bcl-2/Bax (dilution, 1:500); and rabbit anti- $\beta$-actin (dilution, 1:5,000). Subsequent to washing with TBS and $0.1 \%$ Tween (TBST) for $15 \mathrm{~min}$, the membranes were incubated with anti-rabbit $\operatorname{IgG}$ secondary antibodies (dilution, $1: 5,000$ ) at $37^{\circ} \mathrm{C}$ for $1.2 \mathrm{~h}$. Subsequent to washing with TBST for $15 \mathrm{~min}$, detection was performed using an enhanced chemiluminescence kit (KeyGen Biotech Co., Ltd.). Specific bands were quantified using Quantity One 4. 6. 2 (Bio-Rad Laboratories, Inc., Hercules, CA, USA) software. Each experiment was performed in triplicate.

Cell proliferation assay. Cells were incubated in 96-well plates at a density of $1 \times 10^{3}$ cells per well for $24 \mathrm{~h}$ at $37^{\circ} \mathrm{C}$. Cell proliferation was examined on days 1, 2, 3, 4 and 5. Cell Counting Kit-8 (CCK-8) reagent ( $10 \mu$; Dojindo Molecular Technologies, Inc., Kumamoto, Japan) and $90 \mu \mathrm{l}$ of complete medium were added and incubated for an additional $2 \mathrm{~h}$ at $37^{\circ} \mathrm{C}$. Spectrophotometric absorbance at $490 \mathrm{~nm}$ was determined
Table II. Expression of hTRM9L and LIN9 in 70 paired samples of ovarian cancer and ovarian tumor tissues $\left(\chi^{2}\right.$-test).

\begin{tabular}{lccc}
\hline Protein & $\begin{array}{c}\text { Ovarian cancer } \\
\text { tissues }\end{array}$ & $\begin{array}{c}\text { Ovarian tumor } \\
\text { tissues }\end{array}$ & P-value \\
\hline hTRM9L & & & 0.002 \\
Positive & 27 & 45 & \\
Negative & 43 & 25 & 0.028 \\
LIN9 & & & \\
Positive & 29 & 42 & \\
Negative & 41 & 28 & \\
\hline
\end{tabular}

hTRM9L, human transfer RNA methyltransferase 9-like.

with a microplate reader (Bio-Rad Laboratories, Inc.). There were three replicates for each sample.

Flow cytometric analysis. To detect apoptosis, cells were incubated in 6-well plates at a density of $1 \times 10^{5}$ cells per well for $36 \mathrm{~h}$ and subsequentlyre-suspended in 1,000 $\mu \mathrm{l}$ PBS. Annexin V-fluorescein isothiocyanate and 7-amion-actinomyclin D (KeyGen Biotech Co., Ltd., Nanjing, China) dye were added, and samples were incubated at room temperature in the dark for $15 \mathrm{~min}$. Subsequent to passing the cells through a mesh filter, apoptosis was detected using flow cytometry (BD Biosciences, Franklin Lakes, NJ, USA). The cells were counted using CellQuest software FCS2.0 (BD Biosciences), and the data were analyzed using Macquit software FCS 2.0 (BD Biosciences).

For cell cycle analysis, cells were incubated in 6-well plates at a density of $1 \times 10^{5}$ cells per well for $36 \mathrm{~h}$ at $37^{\circ} \mathrm{C}$, and were subsequently re-suspended in $1,000 \mu \mathrm{l}$ of $75 \%$ alcohol overnight at $-20^{\circ} \mathrm{C}$. Subsequent to adding RNAase and PI dye, the cells were incubated at room temperature in the dark for 15 min. Finally, cell cycle analysis was performed using flow cytometry.

Statistical analysis. SPSS 19.0 (IBM SPSS, Armonk, NY, USA) was used for statistical analyses. $\mathrm{P}<0.05$ was considered to indicate a statistically significant difference. Differences in quantitative data between groups were analyzed using Student's t-test, and enumeration data was analyzed using the $\chi^{2}$ test. Associations between the expression levels of hTRM9L and LIN9 were analyzed using Pearson's correlation coefficient.

\section{Results}

Expression of hTRM9L and LIN9 in ovarian cancer tissues. hTRM9L expression was examined in ovarian cancer tissues by IHC. Positive staining of hTRM9L and LIN9 in ovarian cancer tissues was identified in $38.57 \%$ (27/70) and $41.43 \%$ (29/70) of the samples, respectively. However, the rate of positive staining of hTRM9L and LIN9 in ovarian tumor tissues was $61.42 \%(43 / 70 ; \mathrm{P}<0.05)$ and $58.57 \%(41 / 70 ; \mathrm{P}<0.05)$, respectively. The results are shown in Fig. 1 and Table II. A positive correlation was observed between hTRM9L and 

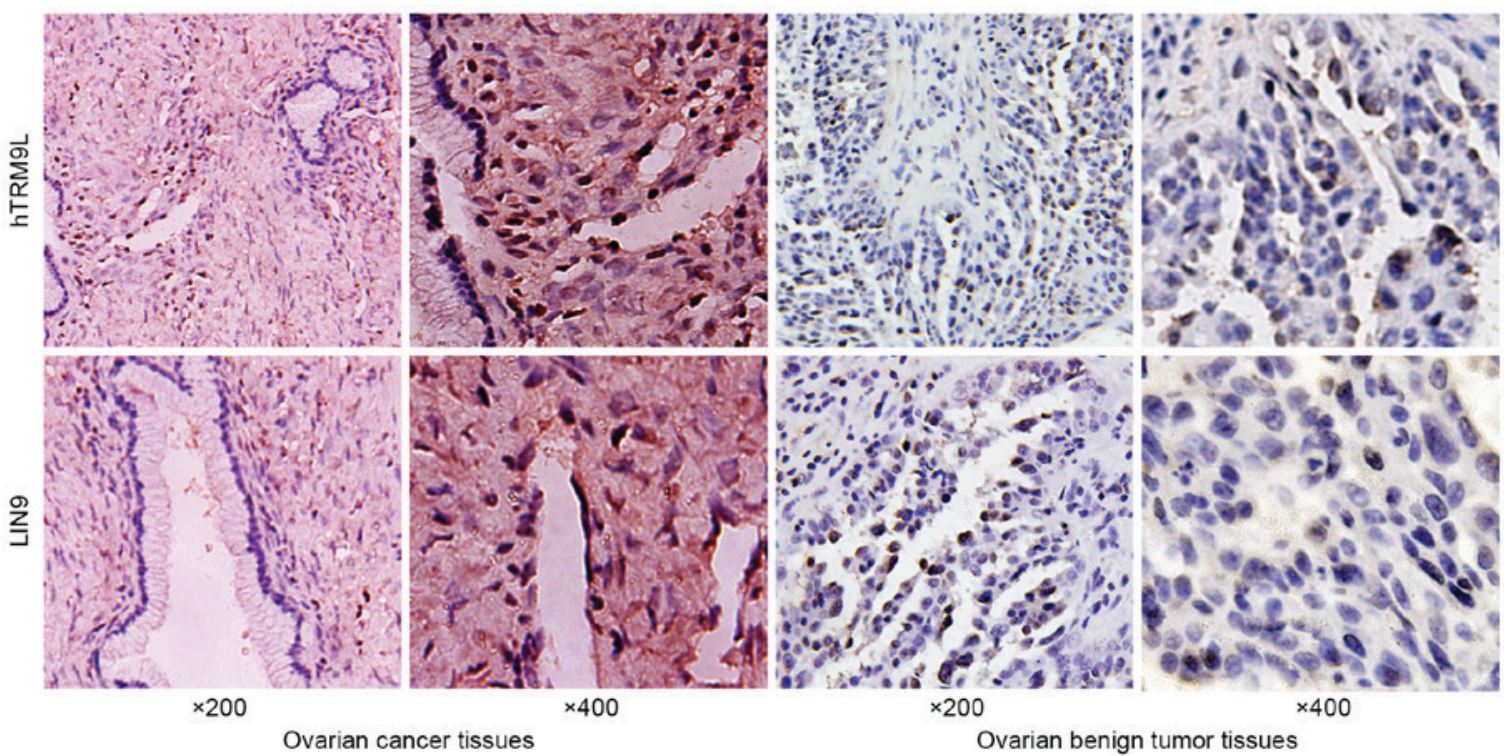

Figure 1. Immunohistochemical staining of hTRM9L and LIN9 in ovarian cancer and normal tissues. hTRM9L, human transfer RNA methyltransferase 9-like.
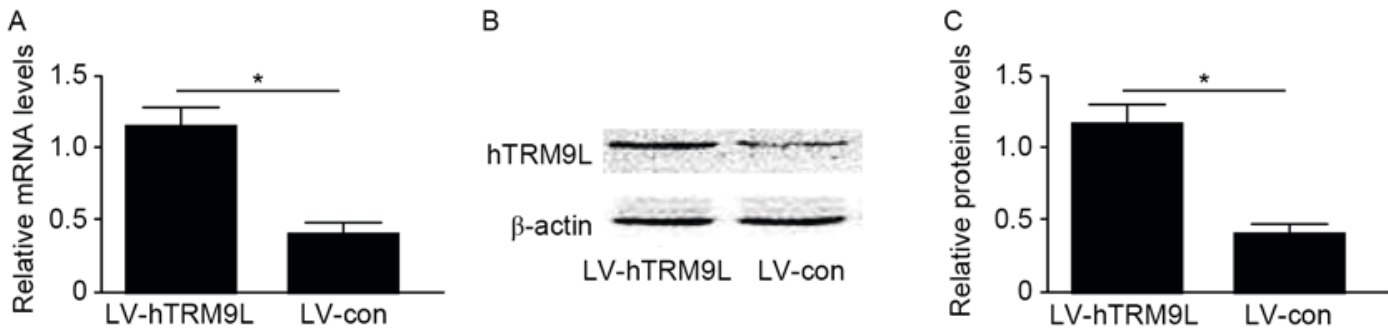

Figure 2. LV-hTRM9L increases hTRM9L expression in HO8910PM cells. (A) hTRM9L mRNA expression in HO8910PM cells transfected with LV-hTRM9L was significantly increased compared with the negative control, as determined by reverse transcription-quantitative polymerase chain reaction. (B) hTRM9L protein expression was increased in cells transfected with LV-hTRM9L compared with cells transfected with the negative control, as shown by western blot analysis and (C) quantification. "P<0.05. hTRM9L, human transfer RNA methyltransferase 9-like; LV, lentiviral; LV-con, negative control group.

LIN9 expression, according to the Pearson's correlation coefficient $(\mathrm{r}=0.406 ; \mathrm{P}<0.05$; Table III). The results indicated that hTRM9L expression is associated with LIN9 expression in ovarian cancer.

hTRM9L promotes LIN9 and Bax expression and inhibits Bcl-2 expression in ovarian cancer cells. Ectopic hTRM9L-expressing ovarian cancer cells (HO8910PM) were used to examine whether hTRM9L promotes LIN9 and Bax expression and inhibits $\mathrm{Bcl}-2$ expression in ovarian cancer cells. hTRM9L expression was induced in HO8910PM cells using a lentivirus (LV-hTRM9L). The expression of hTRM9L at them RNA and protein levels in cells transfected with the LV-hTRM9L was significantly increased compared with the negative control (LV-con), as confirmed by RT-qPCR and western blot analysis, respectively ( $\mathrm{P}<0.05$; Fig. 2). The LIN9 axis performs a critical role in hTRM9L regulation of ovarian cancer cell proliferation and apoptosis. As a result, the increase in LIN9 expression triggered upregulation of pro-apoptotic Bax expression and downregulation of anti-apoptotic Bcl-2 expression. The results indicated that hTRM9L over expression accelerate sovarian cancer cell apoptosis. The LIN9 and $\mathrm{Bax} / \mathrm{Bcl}-2$ expression levels were assayed by RT-qPCR and western blot analysis $(\mathrm{P}<0.05$; Fig. 3).
Table III. Correlation between hTRM9L and LIN9 expression in ovarian cancer tissues.

\begin{tabular}{lcccc}
\hline & \multicolumn{2}{c}{ hTRM9L } & & \\
\cline { 2 - 4 } LIN9 & $\begin{array}{c}\text { Positive } \\
(\mathrm{n}=27)\end{array}$ & $\begin{array}{c}\text { Negative } \\
(\mathrm{n}=43)\end{array}$ & $\begin{array}{c}\text { Pearson's } \\
\text { correlation }\end{array}$ & P-value \\
\hline Positive $(\mathrm{n}=29)$ & 18 & 11 & 0.406 & 0.001 \\
Negative $(\mathrm{n}=41)$ & 9 & 32 & & \\
\hline
\end{tabular}

Effect of ectopic hTRM9L expression on apoptosis, cell cycle and growth of ovarian cancer cells. As shown by flow cytometry, the number of apoptotic HO8910PM cells transfected with LV-hTRM9L was significantly increased compared with the negative control group (LV-con; $\mathrm{P}<0.05$; Fig. 4A). Therefore, hTRM9L may promote HO8910PM cell apoptosis. As shown by CCK-8, the viability of HO8910PM cells transfected with hTRM9L was significantly reduced compared with the negative control group (LV-con; $\mathrm{P}<0.05$; Fig. 4B). The results indicated that hTRM9L inhibits HO8910PM cell growth. As shown by flow cytometry, the proportion of G1 phase HO8910PM cells transfected with LV-hTRM9L was 

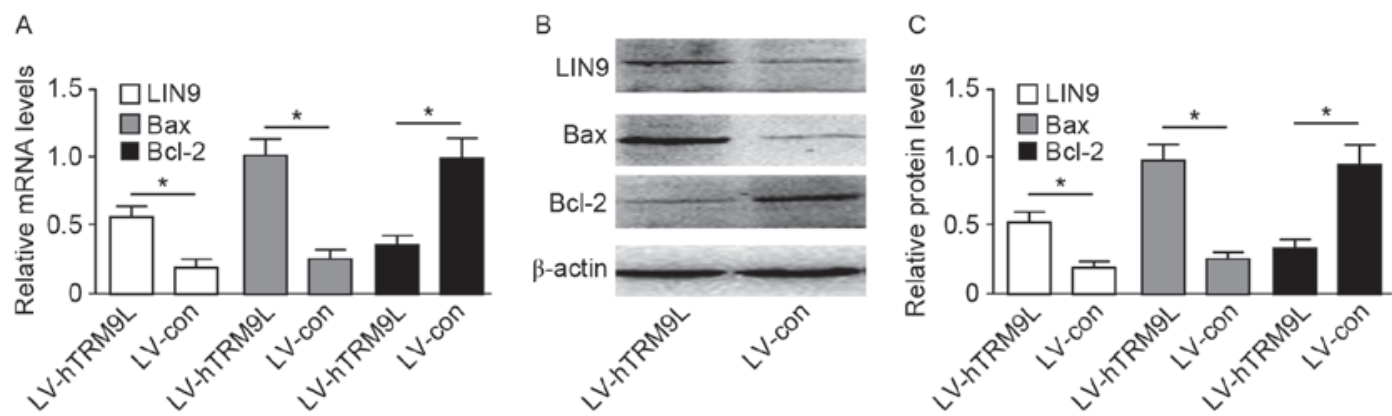

Figure 3. LIN9 and Bax/Bcl-2 expression in LV-hTRM9L-HO8910PM cells. (A) LIN9 and Bax/Bcl-2 mRNA expressionin LV-hTRM9L-HO8910PM cells, as determined by reverse transcription-quantitative polymerase chain reaction. (B) LV-hTRM9L-HO8910PM protein expression in LV-hTRM9L-HO8910PM cells, as shown by western blot analysis and (C) quantification. "P<0.05. hTRM9L, human transfer RNA methyltransferase 9-like; Bcl-2, B-cell lymphoma 2; Bax, Bcl-2 associated X protein; LV, lentiviral; LV-con, negative control group.

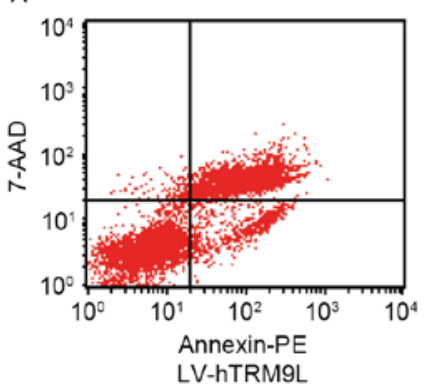

LV-hTRM9L

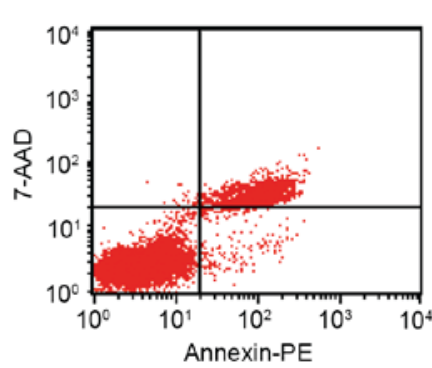

LV-con

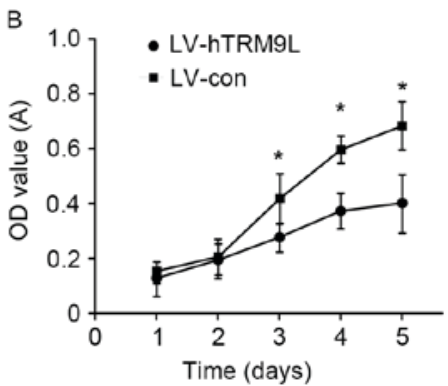

C

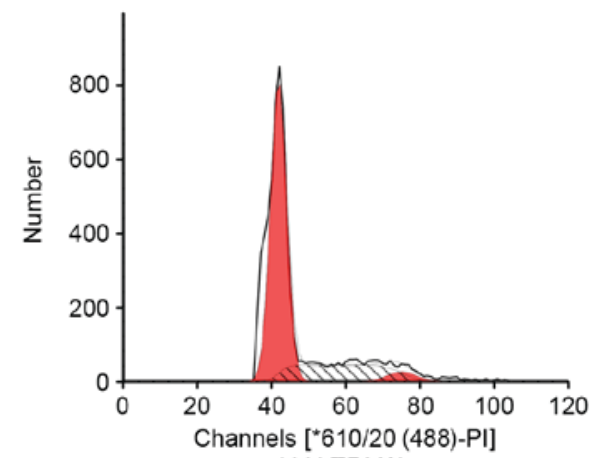

LV-hTRM9L

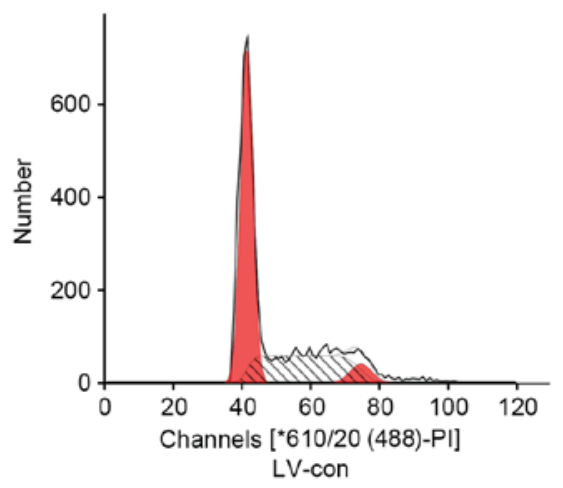

Figure 4. Effects of the ectopic expression of hTRM9L on apoptosis, cell cycle and growth of ovarian cancer cells. (A) Flow cytometry. The amount of apoptosis in HO8910PM cells transfected with LV-hTRM9L was significantly increased compared with the negative control group. (B) Cell Counting Kit-8 assay. HO8910PM cells transfected with hTRM9L exhibited significantly reduced growth compared with the negative control group. (C) Flow cytometry. The percentage of LV-hTRM9L-HO8910PM cells in the G1 phase was increased compared with the negative control group. "P<0.05. hTRM9L, human transfer RNA methyltransferase 9-like; LV, lentiviral; LV-con, negative control group; OD, optical density; 7-ADD, 7-aminoactinomycin D.

significantly increased compared with the negative control group $(\mathrm{P}<0.05$; Fig. 4C).

\section{Discussion}

Ovarian cancer remains the primary threat to the health of women, and its incidence increases annually (1). Therefore, it is necessary to investigate new molecular pathways to counteract this threat. In the present study, hTRM9L expression was depressed in ovarian cancer tissues and significantly associated with LIN9 ( $\mathrm{r}=0.406 ; \mathrm{P}<0.05$ ). To the best of our knowledge, hTRM9L expression was detected in ovarian cancer tissues for the first time, and it was confirmed that the expression of hTRM9L was low.
LIN9, a member of the dimerization partner, RB-like, E2F and multi-vulval class B (DREAM) complex, acts as a tumor suppressor (12). The present study validated that LIN9 transcription and protein levels were upregulated $\sim 2$-fold in hTRM9L-expressing HO8910PM cells. The DREAM (or LINC) complex is a master regulator of mitotic gene expression during the cell cycle. RNA interference-mediated knockdown of the DREAM subunits in human cells inhibits their proliferation via inhibition of mitotic gene expression. LIN9 performs an important role in pRB and p53 signaling to achieve cancer suppression (13).

DREAM function involves the induction of permanent cell cycle arrest $(14,15)$. Cell cycle arrest in G0/G1 by LIN9 may activate the pRB signaling pathway, as demonstrated by flow 
cytometry. At the same time, the proliferation of HO8910PM cells transfected with hTRM9L was significantly reduced compared with the negative control group. In the present study, the number of apoptotic HO8910PM cells transfected with LV-hTRM9L was significantly increased compared with the negative control group, based on flow cytometry. Additionally, pro-apoptotic Bax expression was found to be upregulated, while anti-apoptotic $\mathrm{Bcl}-2$ expression was downregulated. Bax and Bcl-2 are important signaling molecules in the p53 signaling pathway (16).

It was hypothesized that ectopic hTRM9L-expressing ovarian cancer cells (HO8910PM) activate LIN9 entry into the p53 signaling pathway, thereby increasing apoptosis. Apoptosis is considered an important fail-safe mechanism for the prevention of tumorigenesis $(17,18)$.

Rapid tumor cell proliferation and apoptosis inhibition lead to increased malignancy, resulting in an escape from immune regulation. However, hTRM9L may activate LIN9 to enter the p53 signaling pathway, resulting in increased cell apoptosis, as well as the pRB signaling pathway, reducing proliferation (19).

In conclusion, the present study demonstrated that hTRM9L may prevent tumor growth and promote apoptosis by regulating LIN9. This may be a novel breakthrough for ovarian cancer treatment, although additional studies investigating the mechanism are required.

\section{References}

1. Siegel R, Ma J, Zou Z and Jemal A: Cancer statistics, 2014. CA Cancer J Clin 64: 9-29, 2014

2. Gay GM, Lim JS, Chay WY, Chow KY, Tan MH and Lim WY: Reproductive factors, adiposity, breastfeeding and their associations with ovarian cancer in an Asian cohort. Cancer Causes Control 26: 1561-1573, 2015.

3. Dinkelspiel HE, Champer M, Hou J, Tergas A, Burke WM, Huang Y, Neugut AI, Ananth CV, Hershman DL and Wright JD: Long-term mortality among women with epithelial ovarian cancer. Gynecol Oncol 138: 421-428, 2015.

4. Letoquart J, van Tran N, Caroline V, Aleksandrov A, Lazar N, van Tilbeurgh $\mathrm{H}$, Liger D and Graille M: Insights into molecular plasticity in protein complexes from Trm9-Trm112 tRNA modifying enzyme crystal structure. Nucleic Acids Res 43 10989-11002, 2015.

5. Begley U,Dyavaiah M,Patil A, Rooney JP,DiRenzo D, Young CM, Conklin DS, Zitomer RS and Begley TJ: Trm9-catalyzed tRNA modifications link translation to the DNA damage response. Mol Cell 28: 860-870, 2007.
6. Kalhor HR and Clarke S: Novel methyltransferase for modified uridine residues at the wobble position of tRNA. Mol Cell Biol 23: 9283-9292, 2003.

7. Wiseman EF, Chen X, Han N, Webber A, Ji Z, Sharrocks AD and Ang YS: Deregulation of the FOXM1 target gene network and its coregulatory partners in oesophageal adenocarcinoma. Mol Cancer 14: 69, 2015.

8. Flanagan JM, Healey S, Young J, Whitehall V, Trott DA, Newbold RF and Chenevix-Trench G: Mapping of a candidate colorectal cancer tumor-suppressor gene to a 900-kilobase region on the short arm of chromosome 8 . Genes Chromosomes Cancer 40: 247-260, 2004.

9. Begley U, Sosa MS, Avivar-Valderas A, Patil A, Endres L, Estrada Y, Chan CT, Su D, Dedon PC, Aguirre-Ghiso JA and Begley T: A human tRNA methyltransferase 9-like protein prevents tumour growth by regulating LIN9 and HIF1- $\alpha$. EMBO Mol Med 5: 366-383, 2013.

10. Bešević J, Gunter MJ, Fortner RT, Tsilidis KK, Weiderpass E, Charlotte Onland-Moret N, Dossus L, Tjønneland A, Hansen L, Overvad K, et al: Reproductive factors and epithelial ovarian cancer survival in the EPIC cohort study. Br J Cancer 113: 1622-1631, 2015.

11. Hauser S, Ulrich T, Wurster S, Schmitt K, Reichert N and Gaubatz S: Loss of LIN9, a member of the DREAM complex, cooperateswith SV40 large T antigen to induce genomic instability and anchorage-independent growth. Oncogene 31: $1859-1868,2012$.

12. Blais A, van Oevelen CJ, Margueron R, Acosta-Alvear D and Dynlacht BD: Retinoblastoma tumor suppressor protein-dependent methylation of histone $\mathrm{H} 3$ lysine 27 is associated with irreversible cell cycle exit. J Cell Biol 179: 1399-1412, 2007.

13. Hauser S, Ulrich T, Wurster S, Schmitt K, Reichert N and Gaubatz S: Loss of LIN9, a member of the DREAM complex, cooperates with SV40 large T antigen to induce genomic instability and anchorage-independent growth. Oncogene 31: 1859-1868, 2012.

14. Stowell KM: DNA testing for malignant hyperthermia: The reality and the dream. Anesth Analg 118: 397-406, 2014.

15. Tan KO, Nielsen AB, Meier BH and Ernst M: Broad-band DREAM recoupling sequence. J Phys Chem Lett 5: 3366-3372, 2014.

16. Yu Z, Wu F, Chen L, Li Q, Wang C, Dong J and Xie SQ: ETME, a novel $\beta$-elemene derivative, synergizes with arsenic trioxide in inducing apoptosis and cell cycle arrest in hepatocarcinoma cells via a p53-dependent pathway. Acta Pharm Sin B 4: 424-429, 2014.

17. Obata F, Tomioka K and Miura M: Transcriptional profiling of apoptosis-deficient Drosophila mutants. Genom Data 2: 254-257, 2014

18. Carper MB, Denvir J, Boskovic G, Primerano DA and Claudio PP: RGS16, a novel p53 and pRb cross-talk candidate inhibits migration and invasion of pancreatic cancer cells. Genes Cancer 5: 420-435, 2014.

19. Zhou Z, Flesken-Nikitin A, Corney DC, Wang W, Goodrich DW, Roy-Burman P and Nikitin AY: Synergy of p53 and Rb deficiency in a conditional mouse model for metastatic prostate cancer. Cancer Res 66: 7889-7898, 2006. 